\title{
EVALUACIÓN CLÍNICA DE MENINGIOMA INTRAVENTRICULAR: REPORTE DE CASO PACIENTE PEDIÁTRICO Y PACIENTE ADULTO.
}

\author{
DEVI LAKHLANI - OTC1, ANDRES MAURICIO ALVAREZ PINZON M.D., MSc, Ph.D.c ${ }^{2}$ \\ ${ }^{1}$ Duke University School of Medicine \\ Durham, N.C. US. \\ ${ }^{2}$ Médico Cirujano, Magister en Bioética e Investigación \\ Clinica en Neurocirugia - Neurooncologia, \\ Investigador y Coordinador Clinico, \\ Biotechnology Department, Jhon's Hopkins University \\ Candidato a PhD en Neurociencias, Division de Neurociencias, \\ Universidad Salamanca-España \\ Correspondencia: andresmauricioalvarez07@gmail.com
}

Recibido: 12 Noviembre 2016 Aceptado: 3 Febrero 2017

\begin{abstract}
RESUMEN
Los Meningiomas intraventriculares (IVM) representan el 0,5-5\% de todos los meningiomas, de los cuales aproximadamente un $80 \%$ se localizan en el trígono del ventrículo lateral. Se originan en los ventrículos del tope células aracnoideas contenidos en el plexo coroideo constituyen el grupo tumoral intracraneal frecuente en adultos. En la mayor parte de los casos, se trata de tumores histológicamente benignos, de crecimiento lento. Los meningiomas primarios intraventriculares suponen entre el 0,5 y el $3 \%$ de los meningiomas intracraneales. No son comunes en población pediátrica ya que comprenden menos del $3 \%$ de los tumores cerebrales pediátricos y sólo 1.5 a $1.8 \%$ de todas las neoplasias intracraneales. Los tumores pediátricos también muestran una asociación con neurofibromatosis tipo 2 y la exposición anterior a radiación.
\end{abstract}

Se presenta el contraste de dos casos de meningioma intraventriculares en paciente pediátrico y adulto. La edad y el sitio de la lesión en paciente pediátrico es poco común. La escisión de la lesión y tratamiento pronto permitió la cesación de los síntomas en los 2 pacientes.

Palabras clave: Meningioma intraventricular (IVM); Pediatría; Meningioma; Quimioterapia; Radiocirugía; Radioterapia; Resonancia magnética. 


\title{
CLINICAL EVALUATION OF INTRAVENTRICULAR MENINGIOMA: CASE REPORT OF ADULT AND PAEDIATRIC PATIENT
}

\begin{abstract}
The intraventricular meningioma (IVM) account for $0.5-5 \%$ of all meningiomas, of which approximately $80 \%$ are located in the trigone of the lateral ventricle. They originate in the ventricles of content arachnoid cap cells in the choroid plexus are the group common intracranial tumor in adults. In most cases, tumors are histologically benign, slow-growing. Primary intraventricular meningiomas account for between 0.5 and $3 \%$ of intracranial meningiomas. They are uncommon in the pediatric population and comprising less than $3 \%$ of pediatric brain tumors and only 1.5 to $1.8 \%$ of all intracranial neoplasms. Pediatric tumors also show an association with neurofibromatosis type 2 and previous exposure to radiation.
\end{abstract}

We present two contrasting cases of intraventricular meningioma in pediatric and adult patients. Age and site of injury in pediatric patients is rare. The excision of the lesion and prompt treatment allowed the cessation of symptoms in 2 patients.

Keywords: intraventricular meningioma (IVM); Pediatrics; Meningioma; Chemotherapy; Radiosurgery; Radiotherapy; MRI.

\section{AVALIAÇÃO CLÍNICA DE MENINGIOMA INTRAVENTRICULAR: ELATO DE CASO E PACIENTE ADULTO E PACIENTE PEDIÁTRICO \\ RESUMO}

A conta intraventricular meningiomas (IVM) em 0,5-5\% de todos os meningiomas, dos quais cerca de $80 \%$ estão situados na trigone do ventrículo lateral. Eles se originam nos ventrículos de células cap aracnóide conteúdo no plexo coróide são o grupo tumor intracraniano comum em adultos. Na maioria dos casos, os tumores são histologicamente benigno, de crescimento lento. Meningiomas intraventriculares primários representam entre 0,5 e $3 \%$ dos meningiomas intracranianos.

Eles são raras na população pediátrica e compreendendo menos de $3 \%$ dos tumores cerebrais pediátricos $e$ apenas 1,5 a $1,8 \%$ de todos os tumores intracranianos. Tumores pediátricos também mostram uma associação coma neurofibromatose tipo 2 e exposição prévia à radiação.

Apresentamos dois casos contrastantes de meningioma intraventricular em pacientes pediátricos e adultos. Idade $e$ local da lesão em pacientes pediátricos é raro. A excisão do tratamento da lesão ea tempo permitido a cessação dos sintomas em dois pacientes.

Palavras-chave: Meningioma intraventricular (IVM); Pediatria; Meningioma; Quimioterapia; Radiocirurgia; Radioterapia; Ressonância magnética. 


\section{Introducción}

Los meningiomas son generalmente tumores benignos y de crecimiento lento, que se originan de la capa de células aracnoideas, más frecuentes en los sitios donde se sitúan las granulaciones aracnoideas (1). Suelen ser diagnosticados en autopsias hasta en un $30 \%$ de pacientes, de forma incidental. Su incidencia se incrementa con la edad, con una media de 42,9 años para el sexo femenino y de 52 años en el sexo masculino.

Los meningiomas son los segundos tumores cerebrales más comunes después de los gliomas y son poco frecuentes en niños. Los meningiomas primarios en el sistema ventricular sin lesiones adjuntas durales son extremadamente raros, pero son una entidad que puede ser bien identificada originada dentro de los ventrículos del cerebro. Se originan en las células aracnoideas incrustadas en el plexo coroideo. A menudo se encuentran en los ventrículos laterales y de vez en cuando en el tercer y cuarto ventrículos. Debido a que el plexo coroideo es más voluminoso en los ventrículos laterales, la incidencia de los meningiomas ventrículo laterales es más alta en comparación con aquellos en de tercer o cuarto ventrículo.

Se presentan las características clínicas, radiológicas y patológicas de meningioma intraventricular en dos pacientes, con edades entre 13 años y 8 años. $\mathrm{Ni}$ el paciente mostró evidencia de asociación con neurofibromatosis o exposición a la radiación anterior.

\section{Embriogénesis}

En la embriogénesis este tumor está en relación con la formación de las tres capas meníngeas (piamadre, aracnoides y duramadre). Las células aracnoideas sintetizan un gran número de glicoproteínas y moléculas de adhesión, que suelen estar relacionadas con el incremento de la fibrogénesis local ante un traumatismo $(2,3)$. Estas células fibrosas se encuentran mayoritariamente en los sitios donde se encuentran las granulaciones aracnoideas su origen a partir de las crestas neurales puede explicar la ocurrencia de este tipo de tumores con contenido de melanocitos (melanosis neurocutánea, melanocitoma meníngeo primario y el melanoma meníngeo maligno). La capa de células aracnoideas o en ambas, tienen baja frecuencia de división celular, pero ello puede ser incrementado por factores exógenos tales como una infección viral, irradiación, traumatismo de cráneo, factores genéticos o estimulación endógena por hormonas o factores de crecimiento (4-6).

\section{Clínica}

El cuadro clínico de este tipo de lesión es variable, dependiendo fundamentalmente de la localización anatómica, el tamaño y el grado de crecimiento (7-9). Es frecuente la aparición de cefalea recurrente con tendencia a la cronicidad, los déficits neurológicos y las convulsiones; todos ellos de progresión lenta. Los principales complementarios utilizados para establecer el diagnóstico incluyen estudios radiográficos simples de cráneo, estudios tomográficos simples y endovenosos, resonancia magnética (RM) multiplanar con técnica de angiografía cerebral. La modalidad quirúrgica de elección es la craneotomía con la mayor reseccion posible de la lesión, según grados de resección establecidos. Existen otras modalidades de tratamiento que van desde la simple observación clínica e imagenológica hasta métodos combinados que incorporan la radiocirugía, la terapia endovascular y los tratamientos coadyuvantes (quimioterapia y radioterapia); estos últimos reservados principalmente para formas malignas resecadas parcialmente $(3,6,7,10,11)$.

\section{Tratamiento quirúrgico}

El objetivo del tratamiento es la extirpación completa, junto con la duramadre afectada que sirve de base de implantación, para evitar las recidivas. Se utilizan tecnologías para facilitar su extirpación (microcirugía, aspirador ultrasónico, coagulación bipolar) (1,3,11-13). En numerosas ocasiones se procede a la embolización preoperatoria de las ramas meníngeas, lo que permite una manipulación quirúrgica más exangüe y con mejor visión de los planos y estructuras a respetar. Pero todo ello puede acarrear morbilidad importante sobre todo en los de seno cavernoso, parte posterior del seno longitudinal, vaina del nervio óptico, esfeno-orbitarios y en placa. Su extirpación puede causar lesión vascular y neuropatías severas (14-16).

En los meningiomas fuertemente adheridos a senos venosos se prefiere la resección subtotal preservando la integridad vascular y neurológica y tratar el tumor residual con radiación o seguimiento por imagen. 


\section{Radiocirugía}

La radiocirugía estereostática es una alternativa importante para dos tipos de meningiomas: aquéllos de pequeño volumen situados en áreas críticas o de mayor dificultad para el acceso quirúrgico, y como una opción complementaria para los meningiomas resecados parcialmente; en ambos casos, el criterio de tratamiento estriba en que los tumores tengan menos de $35 \mathrm{~mm}$ de eje mayor (17-19). En la serie de Kondziolka et al (20-22) sobre 1.045 meningiomas intracraneales en 972 pacientes, tratados a lo largo de 18 años, se observó que con radiocirugía estereostática con gamma knife hay un control tumoral a los cinco años del $93 \%$ en los de grado I (en los tumores sin confirmación histológica es del 97\%). Sin embargo, en los de grado II y III, el control fue del 50 y del $17 \%$, respectivamente. A los 10 años, el control en los meningiomas de bajo grado ascendió al 91\% (el 95\% en los que carecían de histología). No tuvieron ningún caso de tumor radioinducido.

Halasz et al (23-25) publicaron recientemente su experiencia con radiocirugía estereostática protónica en el tratamiento de 51 meningiomas benignos, obteniendo un control de crecimiento a los tres años del $94 \%$, e indicando que esta técnica es más segura y efectiva que la radiocirugía convencional con fotones, ya que permite una mayor homogeneidad de la dosis, una mejor protección de los órganos de riesgo y una menor dosificación sobre el tejido sano (26-28).

La radiocirugía estereostática fraccionada permite, mediante el fraccionamiento, una mejor protección de los tejidos vecinos, por lo que está indicada para meningiomas del nervio óptico o adyacentes a la vía óptica. La radioterapia con intensidad modulada (IMRT) se ha empleado en meningiomas complejos de la base craneal. El estudio de Milker-Zabel et al con 94 pacientes, de los cuales el $61 \%$ recibió diversos tratamientos previos y el $39 \%$ no tenía confirmación histológica $(4,5,29)$.

Diferentes alternativas terapéuticas se han ensayado con vistas a los meningiomas no resecadles. Basados en la presencia de receptores de progesterona, se han realizado algunos estudios reducidos empleando mifepristona. La hidroxiurea se ha utilizado para el tratamiento de meningiomas, basado en su capacidad de producir apoptosis en cultivos celulares (12,31-33). Diversos estudios han mostrado la estabilización en la progresión en pacientes con meningiomas no resecables (34-36).

\section{Pronóstico}

La extensión de remoción quirúrgica es el factor más importante para la recurrencia $(3,4,37,38)$. La recurrencia después de una extensa remoción tumoral es del $11 \%$ al $15 \%$ de los casos, pero es el $29 \%$ cuando la remoción es incompleta (39-41). A 5 años el índice de recurrencia es del $37 \%$ al $85 \%$ después de una remoción parcial. El índice de recurrencia global a 20 años fue de $19 \%$ en una serie de casos y de $50 \%$ en otra $(12,42-44)$.

\section{Casos Clínicos}

\section{Caso Clínico Paciente Pediátrico}

Paciente masculino de 13 años de edad, quien acudió al servicio por presencia de cefalea continua, con aumento de los síntomas en los últimos 4 meses. Asociado refería emesis y ataxia. Padres refieren disminución de la concentración y reporte de bajo desempeño escolar en el último ano. Paciente fue remetido del servicio de neurología pediátrica al cual fue consultado por bajo rendimiento escolar e hiperactividad. La resonancia magnética (RM) (Figura 1) mostró una lesión de masa bien definida, línea media de la fosa posterior, predominantemente hipointensa en T1 y heterogéneamente hiperintensa en T2 con realce intenso de estudio post contraste (Figura 2). Medía $4.3 \times 3.5 \mathrm{cms}$ de tamaño, comprimiendo el cuarto ventrículo que conduce a la dilatación leve de ventrículos laterales, tercero y cuarto. La masa apareció a extenderse a lo largo de la superficie ventricular de la protuberancia y en la cisterna interpeduncular y fue diagnosticado clínicamente y radiológicamente como meduloblastoma. Posterior craneotomía con enucleación completa de la lesión se realizó junto con la citología intra-operatorio.

Histológicamente, el tumor fue reportado como formación por células dispuestas en racimos lobulados con zonas de espirales. Presencia de núcleos ovalados con cromatina fina granular y Actividad mitótica baja. Por inmunohistoquímica, las células mostraron una fuerte positividad para el antígeno de membrana epitelial. El diagnóstico final fue meningioma meningotelial. 


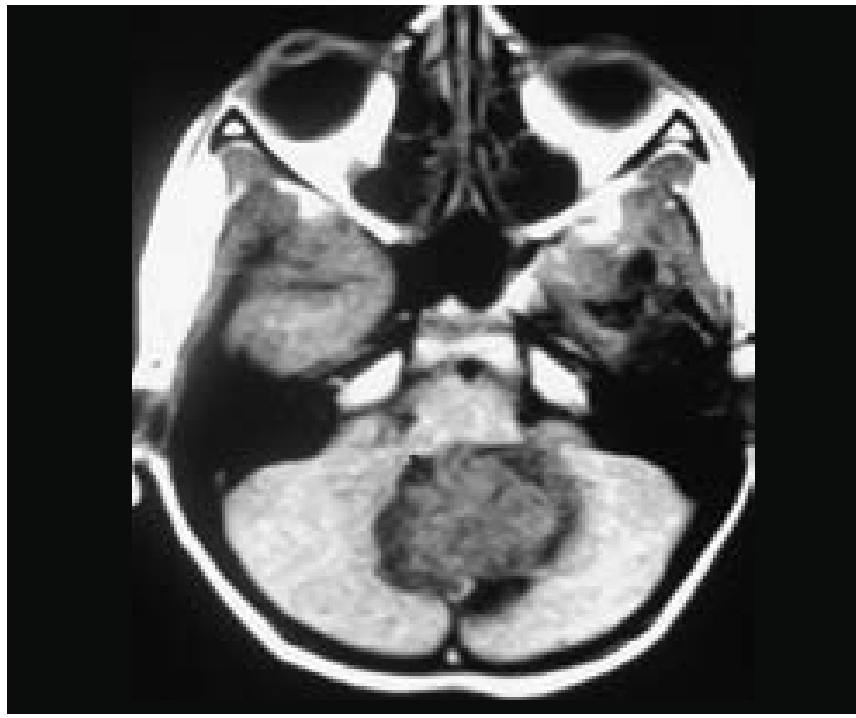

Figura 1. Resonancia magnética cerebral, sin contraste. Imagen en $T 1$ axial: Se evidencia lesión en fosa posterior, ubicada en línea media, con bordes bien definidos.

\section{Caso Clínico Paciente adulto}

El caso corresponde a una mujer de 30 años de edad que consulta al servicio de Neurocirugía por presencia de cefalea sin aura de tipo pulsátil referida a región parietal de 9 meses de evolución. En la evaluación clínica y radiológica a la paciente se le aprecia un síndrome de hipertensión intracraneal y en las pruebas de imagen radiológica muestran una tumoración en el

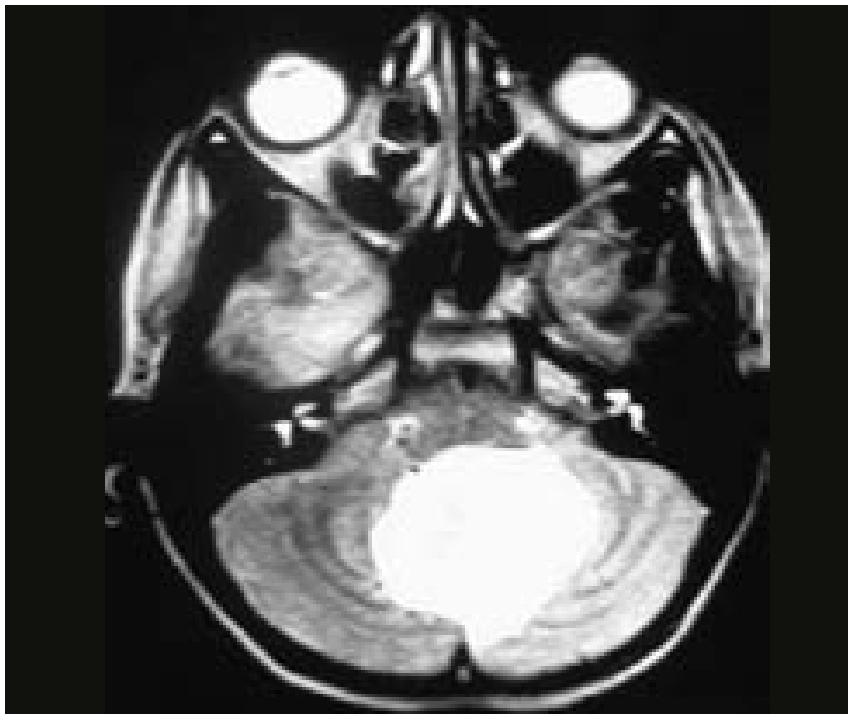

Figura 2. Resonancia Magnética cerebral, Imagen con contraste en T1: Se evidencia reforzamiento intenso de lesión tumoral en la línea media de la fosa posterior.

trígono ventricular izquierdo sin hidrocefalia asociada (figura 3). Tras el diagnóstico, se realiza la extirpación del tumor en su totalidad mediante una craneotomía parieto-occipital izquierda, remitiéndose el tejido para estudio anatomopatológico. Actualmente (18) meses tras la intervención está asintomática, sin evidencia de tumor residual en la TAC, únicamente presenta un déficit campimétrico homónimo derecho ocasionado por la vía quirúrgica utilizada.

\section{Estudios Imagenológicos. Paciente sexo femenino 30 años de edad}

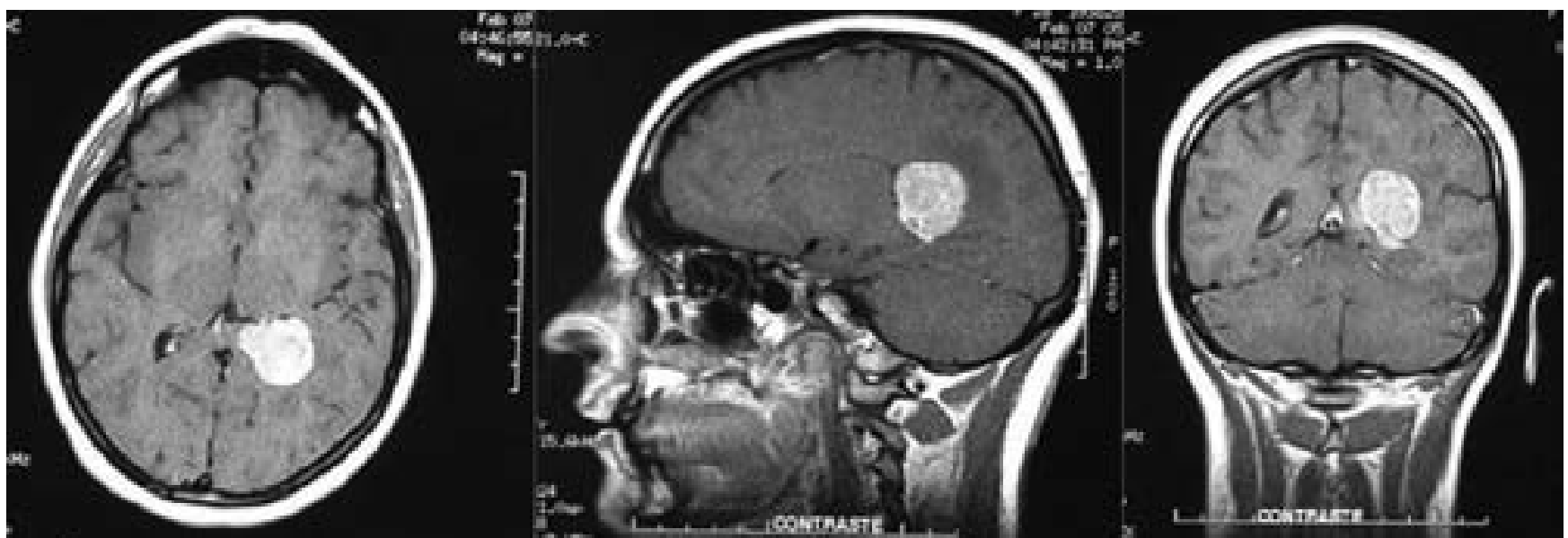

Figura 3. Resonancia nuclear magnética - MRI en secuencia T1 con contraste visualizada en proyecciones axial, sagital y coronal, respectivamente. Se aprecia una imagen hiperdensa, delimitada, que capta contraste de forma homogénea situada en el trígono ventricular izquierdo. 


\section{Hallazgos Anatomopatológicos}

Macroscópicamente, se evidencia una muestra fragmentada de $4 \times 3 \times 1 \mathrm{~cm}$ constituida por porciones de un tejido homogéneo, blanquecino y grisáceo de aspecto fasciculado y consistencia intermedia. Histológicamente, los hallazgos correspondían a un meningioma con-

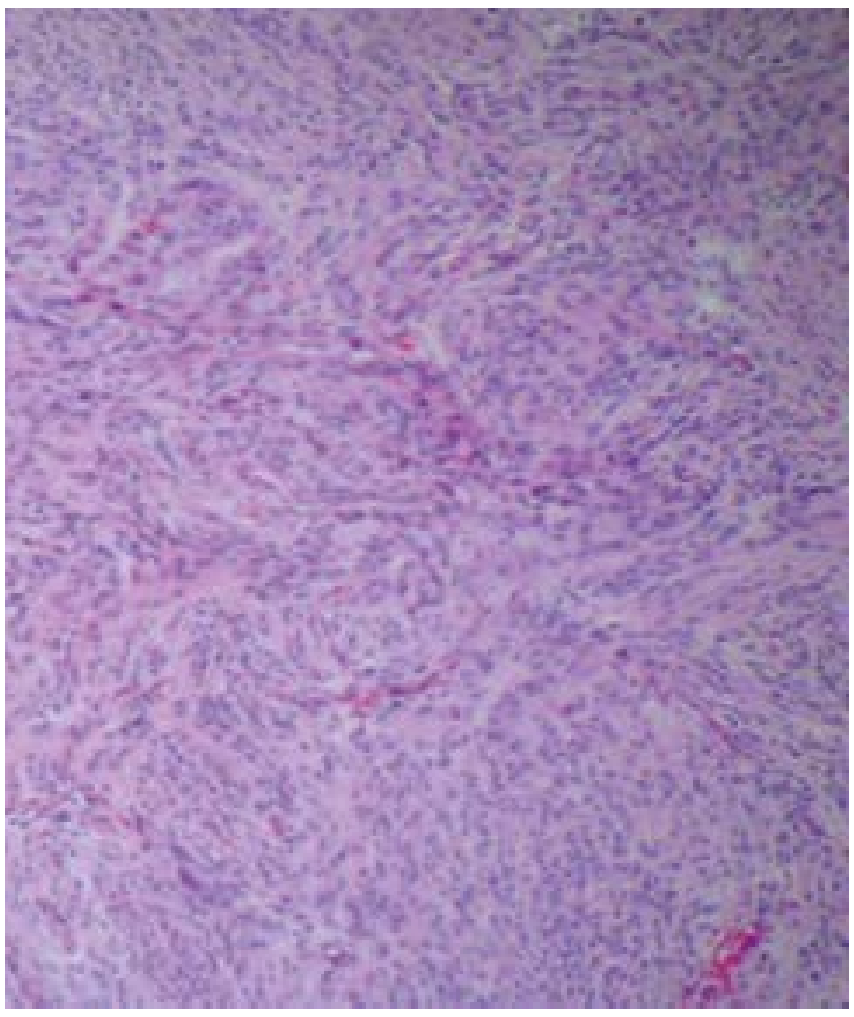

Figura 4. H-E. Aumento original 40X Histología patológica extracción de Tumoración ventricular. -Visión panorámica. Proliferación tumoral de células fusiformes.

\section{Discusión}

Los meningiomas son tumores frecuentes en la población adulta, sin embargo, la localización intraventricular es rara y representa solamente del 0,5 al $3 \%$ de todos los meningiomas (45-47). Surgen de las células aracnoideas de capa (cap cells) que son las células especializadas en granulaciones aracnoideas. Del mismo modo, los meningiomas intraventriculares surgen a partir de células aracnoideas presentes en el plexo coroideo. Es extraña la aparición de meningiomas sin continuidad dural como es el caso de los intraventriculares, sin embargo, parece que estos en particular se originan a partir del vencional de patrón predominantemente meningotelial $(4,9,10)$.

El tumor presentaba una microarquitectura lobular constituida por células eosinofílicas de configuración sincitial, con bordes mal delimitados, y núcleos redondeados u ovales con nucléolos poco prominentes (Figura 4,5).

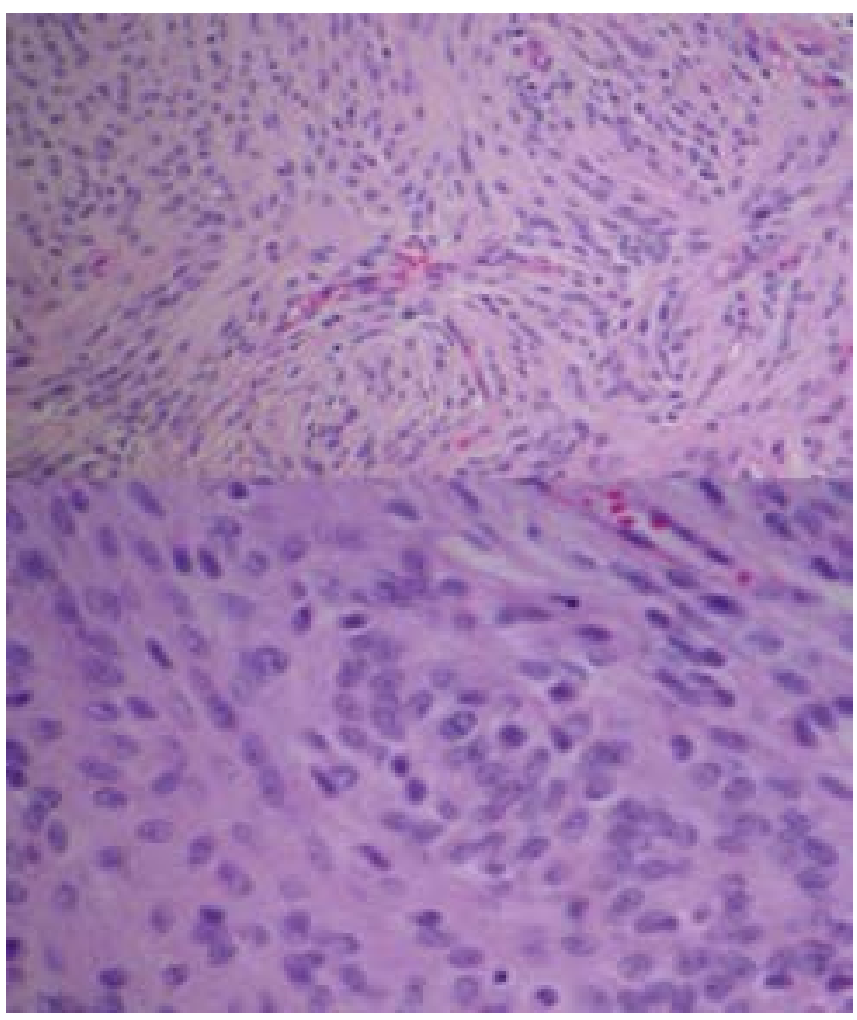

Figura 5. H-E. Aumento original 400X y 100X - Células de aspecto benigno dispuestas en un patrón sincitial

o benigno dispuestas en un patrón sincitial.

estroma aracnoideo que existe en los plexos coroides, que predomina las dos terceras partes en el trígono. La predilección de estos tumores en el ventrículo es por el lado izquierdo con razón de 3:17 lo que resulta aún más raro en el caso presentado en este trabajo cuya localización era derecha(1,4,12,48).

Los meningiomas pediátricos difieren de sus homólogos adultos por su preponderancia de tipo masculino, atipicidad de la ubicación, las tasas más altas de transformación maligna, la recurrencia y la asociación con neurofibromatosis. 
Los tumores intraventricular pediátricos tienen un crecimiento lento lo cual permite una detección a tiempo antes de la evidencia de síntomas complejos.

Los ventrículos cerebrales proporcionan espacio para la expansión y su detección clínica solo puede evidenciarse hasta que las vías del líquido cefalorraquídeo son ocluidlas de forma mecánica, las manifestaciones clínicas al inicio son leves y no específica. Cushing y Eisenhardt (10-12) describen cinco características clínicas de los tumores del ventrículo lateral las cuales cito a continuación:

1. Síntomas de presión con dolor de cabeza ipsilateral,

2. Decisión contralateral mácular e hemianopsia homónima;

3. Contralateral paresia sensoriomotora, con mayor marcación de la afectación sensorial y entumecimiento de las ramas de distribución del trigémino,

4. La evidencia de síntomas de afección cerebelar en más de la mitad de los pacientes,

5. Parexia compleja, dependiendo del estado de compresión del tumor

El cuadro clínico presentado en el contexto pediátrico es debido al aumento de la presión intracraneal, y en general es evidenciado en estos tipos de tumores en cualquiera que sea su ubicación. El paciente consulta por dolor de cabeza, convulsiones del lóbulo temporal y el déficit del campo visual son comúnmente vistos $(5-7,11,20)$.

Los signos cerebelosos y características hipotalámicas con o sin endocrinopatía pueden ser vistos en tumores de 4to y $3 e r$ ventrículo. La neuroimagen es necesaria para el diagnóstico.

Los tumores intraventriculares pueden ser densamente calcificado y visible en radiografía de cráneo en algunas ocasiones, pero no se debe olvidar que el standard de Dx es por medio de la tomografía cerebral y resonancia magnética $(3,49,50)$.

La tomografía computarizada (TC) pueden mostrar calcificación, hidrocefalia obstructiva, y el contraste uniforme en aumento, pero la RM es necesaria para el diagnóstico. La tumoración es hipo o isointenso en T1 y mejora la densidad y de manera uniforme después de la adición de gadolinio. La TC y la RM muestran realce intenso uniforme después de la inyección de contraste los medios de comunicación debido a su falta de sangre $y$ la barrera del cerebro. La histopatología de estos tumores puede ser de cualquier tipo (predominantemente fibroso, fibroblástica, meningotelial, o psamomatoso) tal como se define por la Organización Mundial de la Salud y la Clasificación 's de meningiomas. Generalmente, se diagnostican con base en características morfológicas solamente.

En el caso de meningioma en adultos el abordaje quirúrgico directo con resección total de la lesión es el tratamiento de elección, constituyendo en muchos casos un reto quirúrgico. Los meningiomas del trígono pueden ser abordados en su mayoría mediante la vía trans-parietal, y el grado de resección depende del tamaño de la lesión, de su extensión, del compromiso de las estructuras adyacentes, de la magnitud del sangrado durante la resección y de la evolución clínica del paciente durante el transperatorio (51-53). La vía de Kempe15 es la preferida por otros autores y necesita en muchos casos drenaje ventricular post-operatorio durante $48-72$ horas $(5-9,16,17)$. El paciente adulto que se reporta no requirió drenaje postquirúrgico.

A pesar de que los meningiomas intraventriculares son infrecuentes, los ubicados a nivel del trígono tienen la mayor incidencia en la población adulta. Su diagnóstico diferencial debe incluir los gliomas de bajo grado (7$10,17,18)$.

El pronóstico de los pacientes es usualmente bueno, aunque puede presentarse morbilidad postquirúrgica consistente en trastornos cognitivos y del lenguaje, crisis convulsivas y cefaleas ocasionales. La mortalidad es baja. La aplicación de técnicas endoscópicas permite la resección de lesiones intraventriculares con menor morbilidad, sin embargo, estas técnicas pueden verse limitadas en el caso de lesiones de gran tamaño y consistencia firme como es el caso de los meningiomas $(3,4,25)$. Las complicaciones postquirúrgicas más frecuentes son las infecciones, fístula de líquido cefalorraquídeo, defecto motor, complicaciones respiratorias y sangramientos digestivos, nuestra paciente tuvo una evolución satisfactoria.

\section{Conclusión}

Los tumores intraventriculares son tumores poco frecuentes en la población pediátrica que presentan las características clínicas iníciales de hipertensión intracraneal. Sin embargo, pueden ser retirados intactos y sin daños a las zonas circundantes con cirugía programada meticulosa y no muestran recurrencia en el futuro, en la población adulta, aunque el meningioma es un tumor que habitualmente presenta un sencillo diagnóstico histológi- 
co, es importante tener en cuenta que se puede producir en localizaciones alejadas de la duramadre, destacando la afectación del sistema ventricular, especialmente en el trígono de los ventrículos laterales. Los meningiomas intraventriculares a nivel de trígono son infrecuentes y sólo se reportan series de pocos pacientes en la literatura. El abordaje y resección quirúrgica es el método de tratamiento de elección puede ser llevado a cabo exitosamente y en ausencia de complicaciones importantes.

\section{Conflicto de intereses}

Los autores declaran no tener de manera directa o indirecta ningún tipo de conflicto de intereses financieros, académicos o laborales que puedan poner en peligro la validez de este estudio.

\section{Referencias}

1. Maranhão-Filho P, João Campos CS, Lima MA. Intracranial meningiomas in children: Ten-year experience. Pediatr Neurol. 2008;39:415-417.

2. Bhatoe HS, Singh P, Dutta V. Intraventricular meningiomas: Aclinicopathological study and review. Neurosurgical Focus. 2006;20(3):E9.

3. Qin Y, Kanasaki Y, Takasugi M, Shinohara Y, Kaminou T, Kurosaki M, Ogawa T. Primary fourth ventricular meningioma: a case report of an adult male. Clin Imaging. 2012 Jul-Aug;36(4):379-82

4. Tung H, Apuzzo MLJ. In: Meningiomas. Al-Mefty O, editor. New York: Raven Press; 1991. Meningiomas of the third ventric; pp. 583-591.

5. Guidetti B, Delfini R. In: Meningiomas. Al-Mefty O, editor. New York: Raven Press; 1991. Lateral and fourth ventricle meningiomas; pp. 569-587.

6. Erman T, Göcer AL, Erdogan E. Intraventricular meningiomas: a review and report of eight cases. Neurosurg Quarterly. 2004;14:154-160.

7. Lapras C, Deruty R, Bret PH. Tumors of the lateral ventricles. Adv Tech Stand Neurosurg. 1984;11:103-167.

8. Nakamura M, Roser F, Bundschuh O, Vorkapic $P$, Samii M. Intraventricular meningiomas: a review of 16 cases with reference to the literature. Surg Neurol. 2003;59:491-504.

9. Rushing EJ, Olsen C, Mena H, Rueda ME, Lee YS, Keating RF, Packer RJ, Santi M. Central nervous system meningiomas in the first two decades of life: a clinicopathological analysis of 87 patients. J Neurosurg. 2005;103:489-495.10. 9.

10. Arivazhagan A, Devi BI, Kolluri SV, Abraham RG, Sampath S, Chandramouli BA. Pediatric intracranial meningiomas--do they differ from their counterparts in adults? Pediatric Neurosurg. 2008;44:43-48.

11. Cushing H, Eisenhardt L. Springfield,IL: Charles C
Thomas; 1938. Meningiomas. Their classification, regional behavior, life history and surgical end results. pp. 139-149.

12. Tew JM, Larson JJ. , JrIn: Operative Neurosurgery. Kaye $\mathrm{AH}$, Black PML, editors. London:Churchill Livingstone: 2000. Intraventricular meningiomas; pp. 575-585.

13. Bhatoe HS, Gill HS, Sreeram MN. Densely calcified lateral ventricular meningioma. Ind $\mathrm{J}$ Radiol Imaging. 1994;4:117-118.

14. McDermott MW. Intraventricular meningiomas. Neurosurg Clin N Am. 2003;14:559-569.

15. Kornienko VN, Pronin IN. Tumors of meninges. In: Diagnostic neuroradiology. Berlin, Heidelberg: Springer; 2009;p. 617-805

16. Bertalanffy A, Roessler K, Koperek O, Gelpi E, Prayer D, Neuner M, et al. Intraventricular meningiomas: a report of 16 cases. Neurosurg Rev.2006;29:30-35

17. Pichierri A, Ruggeri A, Morselli C, Delfini R. Fourth ventricle meningiomas: a rare entity. $\mathrm{Br}$ Neurosurgery. 2011;25:454-458

18. Alver I, Abuzayed B, Kafadar AM, Muhammedrezai S, Sanus GZ, Akar ZI. Primary fourth ventricular meningioma: case report and review of the literature. Turk Neurosurg. 2011;21:249-253

19. Osborn AG. Brain tumors and tumorlike masses: classification and differential diagnosis. In: Diagnostic neuroradiology. St. Louis: Mosby; 1994;p. 401-670

20. Elster AD, Challa VR, Contento JC, Elster AD, Challa VR, Gilbert TH, et al.

Meningiomas. MR and histopathologic features. Radiology.1989;170:857-862

21. Akimoto J, Sato Y, Tsutsumi M, Haraoka J. Fourth ventricular meningioma in an adult-case report. Neurol Med Chir (Tokyo). 2001;41:402-405

22. Quadery FA, Okamoto K. Diffusion-weighted MRI of haemangioblastomas and other 2003;45:212219 cerebellar tumours. Neuroradiology.

23. Kornienko VN, Pronin IN. Infratentorial tumours. In: Diagnostic neuroradiology. Berlin, Heidelberg: Springer; 2009;p. 617-718

24. Yamasaki F, Kurisu K, Satoh K, Arita K, Sugiyama K, Ohtaki M, et al. Apparent diffusion coefficient of human brain tumors at MR imaging. Radiology. 2005;235:985991

25. Hakyemez B, Yildirim N, Gokalp G, Erdogan C, Parlak $M$. The contribution of diffusion-weighted MR imaging to distinguishing typical from atypical meningiomas. Neuroradiology. 2006;48:512-520

26. Server A, Kulle B, Maehlen J, Josefsen R, Schellhorn $\mathrm{T}$, Kumar T, et al. Quantitative apparent diffusion coefficients in the characterization of brain tumors and associated peritumoral edema. Acta Radiol. 2009;50:682-689

27. Domingues P, Teodósio C, Ortiz J, Sousa P, Otero A, Maillo A, Bárcena P, García-Macias MC, Lopes MC, de 
Oliveira C, Orfao A, Tabernero MD. Immunophenotypic Identification and Characterization of Tumor Cells and Infiltrating Cell Populations in Meningiomas. Am J Pathol. 2012 Sep

28. Samii M, Gerganov V, Giordano M, Samii A. Two step approach for surgical removal of petroclival meningiomas with large supratentorial extension. Neurosurg Rev 2011; 34: 173-9.

29. García-Navarrete E, Sola RG. Aspectos clínicos y quirúrgicos de los meningiomas de la base del cráneo. III. Meningiomas de la fosa posterior. Rev Neurol 2002; 34: 714-23.

30. De Divittis E, Esposito F, Cappabianca P, Cavallo LM, De Divittis O, Esposito I. Endoscopic transnasal resection of anterior cranial fossa meningiomas. Neurosurg Focus 2008; 25: e8.

31. Ganz JC, Reda WA, Abdelkarim K. Gamma knife surgery of large meningiomas: early response to treatment. Acta Neurochirur (Wien) 2009; 151: 1-8.

32. Kondziolka D, Mathieu D, Lunsford LD, Martin JJ, Madhok R, Niranjan A, et

al. Radiosurgery as definitive management of intracranial meningiomas. Neurosurgery 2008; 62: 53-8.

33. Halasz LM, Bussière MR, Dennis ER, Niemierko A, Chapman PH, Loeffler JS, et al. Proton stereotactic radiosurgery for the treatment of benign meningiomas. Int J Radiat Oncol Biol Phys 2010.

34. Adler JR Jr, Gibbs IC, Puataweepong P, Chang SD. Visual field preservation after multisession cyberknife radiosurgery for perioptic lesions. Neurosurgery 2006; 59 : 244-54.

35. Milker-Zabel S, Zabel-Du Bois A, Huber P, Schlegel W, Debus J.Intensity-modulated radiotherapy for complex-shaped meningioma of the Skull base: long-term experience of a single institution. Int $\mathrm{J}$ Radiat Oncol Biol Phys 2007; 68: 858-63.

36. Clark BG, Candish C, Vollans E, Gete E, Lee R, Martin $\mathrm{M}$, et al. Optimization of stereotactic radiotherapy treatment delivery technique for a base-of-skull meningioma's. Med Dosim 2008; 33: 239-47.

37. Tena-Suck ML, Collado-Ortiz MA, Salinas-Lara C, García-López R, Gelista N, Rembao-Bojorquez D. Chordoid meningioma: a report of ten cases. J Neurooncol 2010; 99 (1): 41-8.

38. Deb P, Sahani H, Bhatoe HS, Srinivas V. Intraventricular cystic meningioma. J Cancer Res Ther 2010; 6 (2): 218-20.

39. Gelabert-González M, García-Allut A, BandinDieguez J, Serramito- García R, Martínez-Rumbo R. Meningiomas of the lateral ventricles. A review of 10 cases. Neurocirugia (Astur). 2008; 19 (5): 427-33.
40. $\mathrm{Li} \mathrm{XZ,} \mathrm{Zhao} \mathrm{JZ.} \mathrm{Operation} \mathrm{of} \mathrm{lateral} \mathrm{ventricular}$ meningiomas of the trigone. Zhonghua Yi Xue Za Zhi 2006; 86 (33): 2321-3.

41. Nayar VV, DeMonte F, Yoshor D, Blacklock JB, Sawaya $\mathrm{R}$. Surgical approaches to meningiomas of the lateral ventricles. Clin Neurol Neurosurg 2010; 112 (5): 400-5.

42. Mahore A, Chagla A, Goel A. Seeding metastases of a benign intraventricular meningioma along the surgical track. J Clin Neuroscienc 2010; 17 (2): 253-5.

43. Eriks Lusis, David H Gutmann. Meningioma: an update. Current Opinion in Neurology 2004; 17: 687-692.

44. Perry A, Scheithauer BW, Stafford SL, Abell-Aleff PC, Meyer FB. "Rhabdoid" meningioma: an aggressive variant. Am J Surg Pathol 1998; 22: 1482-90.

45. Fuller CE, Pfeifer J, Humphrey P, Bruch LA, Dehner LP, Perry A.Chromosome 22q dosage in composite exrarenal rhabdoid tumors: clonal evolution or a phenotypic mimic? Hum Pathol 2001; 32: 1102-08.

46. Sadetzki S, Flint-Richter P, Ben-Tal T, Nass D. Radiation-induced meningiomas: a descriptive study of 253 cases. J Neurosurg 2002; 97:1078-82.

47. Phillips LE, Koepsell TD, van Belle G, Kukull WA, Gehrels JA, Longstreth WT Jr. History of head trauma and risk of intracranial meningioma: population based case control study. Neurology 2002; 58: 1849-52.

48. Bhatia S, Sather HN, Pabustan OB, Trigg ME, Gaynon PS, Robison LL. Low incidence of second neoplasms among children diagnosed with acute lymphoblastic leukemia after 1983. Blood 2002; 99:4257-64.

49. Gursan N, Gondogdu C, Albayrak A, Kabalar ME. Immunohistochemical detection of progesterone receptors and the correlation with Ki-67 labelling indices in paraffin-embedded sections of meningioma's. Int $\mathrm{J}$ Neuroscience 2002; 112:463-70.

50. Nagashima G, Asai J, Suzuki R, Fujimoto T. Different distribution of c- myc and MIB-1 positive cells in malignant meningioma's with reference to TGFs, PDGF and PgR expression. Brain Tumor Pathol 2001; 18:15.

51. Murakami M, Hashimotos N, Takahashi Y, Hosokawa Y, Inazawa J, Mineura K. A consistent region of deletion on 1 p36 in meningioma's: identification and relation to malignant progression. Cancer Genet Cytogenetic 2003; 140:99-106.

52. Radiosurgery provides equivalent tumour control to Simpson Grade 1 resection for patients with small to medium size meningiomas. Int $\mathrm{J}$ Radiat Oncol Biol Phys 2003; 55:1000-5.

53. Hostalot C, Carrasco A, Bilbao G, Pomposo I, Garibi JM. Meningiomas de la tienda del cerebelo. Revisión de nuestra casuística. Neurocirugía 2004; 15: 119-27. 